\title{
Implications de la crise de l'environnement et du développement sur la santé et le mouvement migratoire en Afrique
}

\author{
Shally B. Gachuruzi
}

\begin{abstract}
It is well known that environmental and development crises have devastating effects. This article seeks to demonstrate that the implications of those crises have repercussions on health and population migration in Africa. It is concluded that development inappropriate to the environmental conditions results in a series of debilitating conditions for the health of the population. In turn these conditions can trigger further crisis in addition to whatever natural calamities and population increases occurring.
\end{abstract}

\section{Introduction}

Le Sommet de la planète, tenu à Rio en 1992 s'est caractérisé par plusieurs lignes d'orientation. Il s'agit notamment de l'alerte sur les dangers de l'amincissement de la couche d'ozone, de la ménace omniprésente des déchets toxiques, de la pollution, des conséquences de la déforestation, de l'urgent besoin de la protection des écosystèmes rares et fragiles ainsi que de l'insistance sur la relation sinusoïdale entre l'environnement et le développement. Depuis lors, chercheurs, technocrates, politiciens, organismes gouvernementaux et non gouvernementaux, tentent de trouver des voies et moyens susceptibles d'apporter des solutions à ce problème. Le travail abattu jusqu'ici est très remarquable et l'abondance des publications sur la question témoigne éloquemment de l'intérêt qu'on lui porte. Et pourtant, le problème persiste, le monde s'inquiète et s'intérroge. Cet article s'inscrit dans cette perspective de questionnement et vise à démontrer que la crise de l'envi-

Dr. Shally Gachuruzi is a research associate at CRS, York University, with interests in forced migration, the environment and development. ronnement et du développement a des répercussions sur la santé et le mouvement migratoire des populations africaines.

\section{La crise de l'environnement en Afrique}

Selon les études faites par le Groupe $\mathrm{d}^{\prime}$ Etudes et de Recherches sur les Politiques Environnementales (GERPE) la préoccupation de " 1 'environnementen l'Afrique n'est plus un problème importé. La prise de conscience est réelle" (GERPE 1990, 2). Dans la même veine Simard et Djénéba Diarra $(1993,22)$ soulignent que

les pays africains, collectivement, ne sont pas demeurés en marge du débat sur l'environnement. Depuis les années 60 , de nombreuses conférences ont permis une concertation des pays du continent africain sur les questions liées à l'environnement. De manière générale, les préoccupations africaines ont suivi l'évolution du débat à l'échelle mondiale. Dans les années $60-70$, on parlait surtout de la conservation de la nature. La décennie suivante a vue se généraliser la réflexion sur l'environnement. On passe alors de la notion de la conservation à une analyse plus globalisante qui déborde d'une conception essentiellement physique de l'environnement pour inclure les aspects économiques et sociaux de la crise environnementale.

Ces deux études laissent penser cependant que l'intérêt pour la protection environnementale en Afrique est récent, parce que ne remontant que de la période post-coloniale. Un survol rapide des principaux problèmes environnementaux peut donc laisser croire que les africains ignoraient les règles élémentaires de la protection de leur environnement et qu'ils devaient tout apprendre des experts dans ce domaine. Et pourtant, l'histoire a démon- tré qu'à l'instar de peuple d'autres latitudes planétaires, les indigènes africains ont toujours vécu en harmonie avec leur milieu naturel.

Pour les pygmées (peuple primitif) par exemple qui sont restés en marge de la civilisation, la faune et la flore sont sacrées. Le déboisement est quasi interdit et la chasse se fait par nécessité. "The forest is a father and mother to us, (...), like a father or mother, it gives us everything we need, food, clothing, shelter, warmth and affection. Normally everything goes well, because the forest is good with its children, but when things go wrong, there must be a reason" (Thurnbull 1962,260 ).

Ce témoignage illustre comment les pygmées et la nature sont dans une relation de dominés et dominants, dont les dominés sont justement les humains. Cette vision primitive et contraire au positivisme scientifique et à la tradition Judéo-Chrétienne qui veulent que les êtres humains dominent la nature et la transforme à leur guise, commence cependant à influencer la pensée écologique des dernières décennies.

Pour ce qui concerne la flore par exemple, l'Afrique a une tradition intéressante sur le culte des forêts sacrées. C'est dans ces dernières où on pratiquait l'initiation aux jeunes garçons, comme la circoncision, les devoirs et les obligations du jeune homme en voie de devenir adulte. C'est dans ces forêts sacrées aussi qu'on implorait les dieux pour apporter la pluie en cas de sécheresse prolongée et une bonne récolte en cas des disettes. C'est enfin dans ces forêts sacrées qu'onjetait les placentas après les accouchements. Il était donc strictement interdit d'exploiter ces forêts sous peine des sanctions sévères et quiconque grugait ces réserves fami- 
liales sacrées s'exposait aux grands ennuis. Comme on peut le constater, le bien-être et la santé étaient intimement liés à la protection de l'environnement.

Dans le même ordre d'idée, la commission sur les pays en développement et le changement de l'environnement planétaire (CRDI 1992, 47), affirme que les habitants des forêts détruisent rarement leur environnement. C'est l'abattage ou le déchiffrage à caractère commercial qui dégrade les ressources forestières et appauvrit les collectivités qui en vivent. Ils sont ainsi les victimes et non les auteurs d'une crise environnementale en Afrique qui ne s'est manifestée qu'avec la colonisation et qui a coïncidée avec l'ère de l'industrialisation.

Le débat sur cette crise place le Nord comme le champion pollueur, producteur des déchets toxiques et nucléaires et responsable de la diminution de la couche d'ozone. Le Sud pour sa part serait coupable de la déforestation qui cause des perturbations climatiques en entraînant une baisse pluviométrique, de la sécheresse, de l'accélération de la désertification et du réchauffement de la planète.

Nombreux sont cependant des analystes occidentaux qui font de la surpopulation le cheval de bataille de la crise actuelle en Afrique. Le Sud serait ainsi responsable de l'explosion démographique qui fait que les ressources deviennent insuffisantes obligeant la population à s'attaquer aux réserves naturelles. Cette pauvreté les rendrait incapables de mettre en place des politiques visant la sauvegarde de l'environnement.

En Ouganda par exemple, le revenu par habitant a baissé de $28 \%$ entre 1980 et 1990 , au Niger de $24 \%$ et en Zambie de $20 \%$ pour la même période (CRDI 1992, 28). Il nous semble donc utopique de croire que ces gens qui sont aux prises avec la famine puissent inclure la protection de l'environnement dans leurs priorités.

Les agences internationales de développement abondent souvent dans le même sens en indiquant que,

la plupart des pays en développement n'ont pas les moyens d'adopter des politiques réactives ou d'assumer les coûts de mesures rigoureuses de lutte contre la pollution. Il en est donc résulté une détérioration massive de leur environnement et un épuisement à l'échelle mondiale des ressources renouvellables qui sont à la base de tout développement (ACDI 1987, 5).

La situation est d'autant plus dramatique que certains pays de l'Afrique de $1^{\prime}$ Ouest ont déjà perdu plus de $80 \%$ de leur forêt dense en 50 ans. Cette situation va aller de mal en pire au Sahel où la consommation en bois-énergie va être multipliée par trois alors que la superficie forestière va être réduite de $50 \%$ d'ici l'an 2030 (Catinot 1984, 11).

$D^{\prime}$ autres par contre, telle que la commission sur les pays en développement et les changements de l'environnement planétaire, pensent que les arguments qui lient la dégradation du milieu à l'abondance de populations pauvres ont tendance à oublier que c'est dans bien de cas l'aliénation du sol et de ses ressources, souvent au profit des intérêts commerciaux, qui explique la détérioration de l'environnement des collectivités locales.

On ne devrait pas non plus ignorer que pour la population locale, la forte natalité se justifie car elle a une portée sociale et économique. L'explication qui tente de justifier la pauvreté des pays du Tiers Monde par l'explosion démographique pèche donc par réductionisme. Voilà pourquoi, il est important de considérer les liens entre croissance démographique et environnement dans un contexte social plus large car même si les familles nombreuses croissent les contraintes économiques qui s'exercent sur les pauvres, on doit dire que les familles pauvres préfèrent souvent avoir un plus grand nombre d'enfants pour leur sécurité économique. Ainsi, réduire la pauvreté, ce serait aussi diminuer la taille des familles. Les efforts de limitation des naissances sans solution du problème de pauvreté risquent de demeurer infructueux (CRDI 1992, 49).

Comme on peut le constater, on ne peut que dresser un tableau pessimiste pour l'environnement en Afrique. Il convient de souligner que si des actions immédiates ne sont pas amorcées, les efforts de redressement de la situation deviendront de plus en plus onéreux et le nombre des réfugiés de l'environnement et du développement ne fera que s'amplifier. Comment y parvenir quand on sait que la dégradation des ressources renouvelables s'inscrit dans une conception de développement intrinsèquement liée au paradigme occidental de la modernité qui signifie, productivité, efficacité, maximisation des profits, etc?

\section{La crise du développement}

Selon la Commission Brundtland, "le développement durable est un développement qui répond aux besoins du présent sans compromettre la capacité des générations futures de répondre aux leurs" (CMED 1989, 52). Deux notions sont liées à ce type de développement. Celle des besoins "et plus particulièrement, des besoins des plus démunis, à qui il convient d'accorder la plus grande priorité et celle "de limitation que l'état de nos techniques et de notre organisation sociale imposent sur la capacité de l'environnement à répondre aux besoins actuels et à venir" (CMED 1989, 52).

Comme écrit J. Galtung (1977) "la notion des besoins fondamentaux est loin d'être une idée nouvelle. On la retrouve chez Marx, chez Gandhi, au coeur de l'idéologie de la FAO et dans la tradition catholique avec le notre Père et son "donne-nous aujourd'hui notre pain de ce jour". C'est face à la pérsistance et à l'aggravation des problèmes, de la misère et de la faim que Galtung repense l'aide internationale accordée au Tiers Monde en visant tout d'abord les besoins fondamentaux. Il définit ainsi le développement comme étant le développement des êtres humains parce qu'ils sont au centre des priorités de développement.

Derrière ce type de développement dit humain et cette capacité de faire le choix des besoins à satisfaire, on retrace l'importance de l'autonomie et de la pratique de l'auto-développement dite aussi "développement endogène". Ce développement réflète un 
nouveau courant de pensée des organismes internationaux et plus particulièrement de l'UNESCO qui est à la recherche $d$ 'un autre développement. L'endogéniété de ce type de développement se caractérise par une conception ouverte et pluraliste du développement qui respecte la spécificité de chacun. On demeure ainsi ouvert et confiant en l'être humain et dans ses technologies et stratégies nouvelles qu'il élaborera pour arriver à un développement qui lui est approprié. Nous retrouvons dans la pensée de l'UNESCO, les notions de flexibilité, $d$ 'ouverture et de confiance en l'homme et un développement à multiples facettes de Galtung.

Ce modèle de développement endogène ajoute l'auteur, n'est cependant réalisable qu'avec le soutient d'une part, d'un nouvel ordre économique international qui refuse les modèles de développement imposés, extravertis, inadaptés et destructeurs de l'identité culturelle des peuples et d'autre part, d'une politique d'auto-valorisation. En effet, il est important que les sociétés croient en leur valeurs intrinsèques, en leur capacité non seulement de survie et de stabilité, mais aussi en leur propre créativité en vue d'un progrès quin'est pas seulement matériel etéconomique, mais globale, authentique, qualitatif et humain.

On sait cependant aujourd'hui que pour la plupart de pays, le bilan de ces tentatives est nul voire désastreux. Les signes les plus visibles de l'échec sont d'une part, l'endettement public qui est de plus en plus contraignant et $d$ 'autre part $d$ 'une dépendance alimentaire croissante se traduisant par des famines endémiques. Le passé colonial qui a inspiré le modèle de gestion du pouvoir en Afrique a contribué à amplifier la situation. En effet, la période post-colonial a renforcé davantage les structures centralisées justifiant cette position par le souci de revêtir aux jeunes nations des formes unitaires et centralisées. Par conséquent, il était hors de question de cautionner des projets qui tentaient de réduire les différences ethniques, religieuses ou linguistiques.
On comprend alors que la gestion du pouvoir en vue de développement ne pouvait pas profiter à toutes les composantes sociales. En effet, les élites ont vite compris l'intérêt qu'il y avait à maintenir les structures coloniales de la gestion du pouvoir. Celleci consistait principalement en la ponction des revenus fiscaux, à l'obligation de la culture de rente au détriment de la culture vivrière ainsi que du monopole de commercialisation par ses offices. Les paysans sont exclus à la participation du pouvoir de décision alors qu'ils sont directement concernés par le processus même du développement.

Cette méconnaissance de la logique paysanne explique en gros leur résistance qui se manifeste par le refus de coopérer et d'exécuter les ordres qui viennent d'en haut. Au contraire, en participant activement au processus de développement, chaque membre fait sa part et contribue ainsi à améliorer son environnement immédiat. En les associant à l'élaboration et à l'exécution de ces programmes, les individus sentent qu'ils oeuvrent réellement pour leur propre bien. Dans un tel contexte, les individus et leur cadre de vie sont dans une sorte de symbiose capable de produire une alchimie envie du développement.

Aujourd'hui, les Africains sont confrontés à un grand défi; celui de rédynamiser les élans de métamorphose sociale. Le problème qui se pose aux peuples Africains est de savoir comment affronteront-ils ce défi et par quel moyen fertiliseront-ils leurs actions pour qu'ils grandissent et portent des fruits. Le développement, l'espoir de vie féconde et d'un avenir présomptueux sont fonction de la manière dont ce défi sera levé.

L'impératif le plus urgent qui s'impose est $d$ 'amorcer une lutte contre les différentes formes de pauvreté qui les affaiblissent et les asphyxient. Il va donc sans dire que la recherche de l'équilibre entre l'homme et son milieu naturel est un préalable pour minimiser les implications négatives de la crise de l'environnement sur la santé de la population.

\section{La crise de l'environnement et du développement et ses implications sur la santé de la population}

De manière générale, la bonne santé de la population est tributaire à la salubrité de leur environnement immédiat. Selon un spécialiste du développement rural intégré, le risque le plus grave que les familles pauvres courent provient de la pollution de l'eau provoquée non pas par des produits chimiques mais par des matières fécales, non pas par des déchets industriels mais par des déchets humains. C'est pourquoi, ajoute-t-il, le manque d'eau potable et de système d'assainissement sûres est leur problème environnementale le plus aigu, car il les expose aux maladies diarrhéiques, à la bilharziose, à l'ankylostomiase, à la dracunculose, au choléra et à la typhoïde. Les multiples conséquences dues au manque de salubrité sont aggravées par la sous-limentation et la malnutrition qui affectent la santé de la population. Pour cet observateur de la scène africaine, c'est cela la crise silencieuse de l'environnement; celle qui prélève son tribut quotidien sur la vie et la santé de millions de personnes dont la voie mériterait bien d'être entendue dans le débat sur l'environnement. Malheureusement,
la plus grande de toutes les catastro- phes n'est pas transmise sur les écrans de télévision. Il s'agit de la tra- gédie quotidienne des enfants les plus pauvres du monde qui souffrent de malnutrition et de maladies chro- niques. Parce que cela se produit cha- que jour, ce n'est pas un événement. Pourtant jamais famine, jamais inon- dation, jamais guerre n'a tué 250000 enfants en une semaine (UNICEF 1992).

Si dans quelques pays d'Afrique on ne peut pas se plaindre du côté des protéines végétales en cas de bonnes récoltes, tel n'est pas le cas pour les protéines animales et les produits laitiers. La viande et le poisson se mangent rarement et les vaches ne donnent pas suffisamment du lait. Bien plus, ce n'est pas tout le monde qui a des vaches. Le lait qui pourrait être dispo- 
nible est celui des chèvres et des moutons. Or, il n'est pas consommé à cause des tabous qu'entoure ce produit. La production agricole est souvent aussi insuffisante et il n'est pas rare de voir les provisions alimentaires se terminer avant la nouvelle récolte. Comme on le sait, ceci condamne la population à une sous-alimentation qui est déjà doublée de malnutrition.

En se nourrissant rien qu'avec des céréales, comme le sorgho, le mil, riz, mais et les tubercules, comme le manioc, l'igname, la pomme de terre ainsi que des fruits tel que la banane plantain qui n'apportent presqu'exclusivent que de l'amidon, les gens s'exposent aux maladies carencielles comme le Kuashiorkor que l'on trouve dans plusieurs pays d'Afrique.

Selon Sukhatme $(1976,113-40)$, les régimes alimentaires actuels, dans les pays en développement, sont très insuffisants. Vingt pour cent au moins y est sous alimenté, une personne sur trois souffre de malnutrition protéique, plus de la moitié est «démunie». Environ $60 \%$ des habitants des pays en développement souffrent de la sousalimentation et de la malnutrition, voire les deux. Ceci signifie que 1 à 1.5 milliards d'hommes périssent de la faim ou de la malnutrition et que, parmi eux, près de 500 millions n'ont même pas à manger.

Soyed Ahmed Marei estime pour sa part que la malnutrition affecte environ 460 millions d'êtres humains dans le Tiers Monde. Elle exerce une influence désastreuse sur la santé, le développement et la capacité qu'ont les gens de s'instruire et de travailler pour simplement survivre.

Tous les ans, dans le monde en développement, quelques 15 millions d'enfants meurent des suites des infections et de la malnutrition, et donc à un rythme de 40000 enfants chaque jour. Un demi million de femmes meurent de complications associées à la grossesse. Les nouveaux nés qui survivent doivent résister aux assauts répétés de la maladie. Des millions de personnes souffrent de parasitoses, lésions, cécité et troubles graves de divers ordre (CRDI 1992, 17).
Un récente étude (CRDI 1992) indique qu'au Nigeria par exemple, plus de 14 millions de personnes souffrent de la sous-alimentation chroniques, elles sont 12 millions en Ethiopie et plus de $40 \%$ de la population tchadienne. Les effets de mauvais régimes alimentaires sont souvent considérablement accentués par les infections et des contaminations dues au manque des soins et $d$ 'hygiène. Beaucoup d'auteurs affirment qu'il y a une relation de cause à effet entre la pauvreté et la désabilité dans les pays du Tiers Monde. Non seulement les victimes sont les pauvres mais aussi la pauvreté elle-même est la cause principale de la désabilité. Selon eux plusieurs personnes dans la plupart des pays du Tiers Monde sont handicapées. Parmi les principales causes, ils citent le manque de moyens pour mettre en place des politiques de la sauvegarde de l'environnement envie de garantir de la nourriture, l'absence d'une politique énergique en matière de santé, les efforts étant concentrés ailleurs. Le problème est beaucoup plus dramatique chez les femmes enceintes. En effet, quand elles ne mangent pas assez, il en résulte une détérioration irréversible de leur santé affectant aussi gravement les foetus qui courent le risque de naître avec un handicap mental ou physique.

Outre l'absence des latrines, le manque critique d'eau potable et les mauvais régimes alimentaires, un autre effet de la crise de l'environnement sur la santé est provoqué par l'absence de réglementation dans l'emplacement des usines tels que les garages, les menuiseries qu'on retrouve au coeur des quartiers résidentiels. Bruits, poussière, huiles et autres matières dangereuses et polluantes font partie du quotidien de la population qui vit dans ces quartiers.

Aussi, dans plusieurs villes africaines, les hommes et les égouts font bon voisinage. Les caniveaux n'ont reçu la visite des pelles qu'à l'époque coloniale. Au sujet des réseaux d'égouts, l'accumulation d'eaux usées sur le sol et dans les rigoles est non seulement incommodant et crée des risques évi- dents pour la santé, mais elle peut même provoquer des graves épidémies lorsque les collections d'eau sale servent des gîtes aux moustiques". Dans le même ordre d'idée Amadou Ba $(1994,21)$ indique,

l'absence de système efficace de canalisation des eaux de surface ou d'enlèvement des ordures ménagères demeurent une des principales caractéristiques de nombre de villes du Tiers Monde. Là, l'approvisionnement en eau potable constitue sans nul doute un des indicateurs les plus révélateurs des inégalités à l'intérieur de l'espace urbain. (...) pour la grande majorité de la population entassée dans les quartiers les plus pauvres de la ville ou les adductions d'eau sont totalement inexistantes, l'approvisionnement en eau potable représente une véritable corvée. En saison d'hivernage, la récupération des eaux de ruissellement à partir des toits en tôles ondulées des maisons représente un type d'approvisionnement en eau, assez courant malgré tous les risques de maladies qu'il comporte (...)

L'auteur s'appuit sur les résultats d'une enquête réalisée au Sénégal et au Mali portant sur les relations entre le mode d'urbanisation et la transmission du choléra pour démontrer que seulement le tiers des maisons des personnes touchées par la maladie vivait dans des conditions hygiéniques déplorables. Au Sénégal par exemple, le réseau d'égout de la ville reste très aléatoire: des canalisations bouchées, des égouts à ciel ouvert, des latrines domestiques le plus souvent dans un état $\mathrm{d}$ 'hygiène de fonctionnement totalement défectueux. Selon l'auteur, Bamako/Mali, une ville de près de 650 000 habitants déverse annuellement plus de cent milles tonnes d'ordures, pour n'enlever qu'un peu plus du tiers des déchets. Au plan de l'assainissement, la ville ne possède pratiquement aucun système tout à l'égout ni autres équipements collectifs.

Un autre impact négatif de la crise de l'environnement et du développement provient de grands barrages. Selon Chantal Euzéby $(1979,773)$, l'eau, facteur indispensable à la survie de l'homme et de son environnement

Refuge, Vol. 15, No. 2 (April 1996) 
peut aussi être un obstacle au progrès économique et social lorsqu'elle permet la propagation et l'extension de certaines maladies comme la bilharziose, et le paludisme. Elle cite le cas de grands travaux hydrauliques qui sont responsables de l'hébergement des vecteurs des maladies parasitaires en soulignant que les projets d'aménagement pour une utilisation plus rationnelle de l'eau à des fins agricoles, industrielles ou humâmes (usages domestiques, hygiène, ...) ont modifié le fragile équilibre sol-eau-climat des pays en voie de développement et ont souvent conduit à une aggravation de leurs problèmes sanitaires.

L'eau peut altérer la santé et transmettre des maladies soit parce qu'elle héberge les vecteurs ou hôtes intermédiaires des maladies parasitaires et virales telles que le paludisme, fièvre jaune, bilharziose, soit parce qu' elle contient et transporte des micro-organismes porteurs des maladies contagieuses (choléra ou dysenterie par exemple) soit encore parce qu'elle reçoit et achemine les produits chimiques pouvant occasionner des sérieux troubles sur la santé de l'homme (Idem).

En ce qui concerne la bilharziose, l'auteure a constaté que dans certaines localités proches du lac Volta au Ghana, le taux ou la prévalence de la bilharziose vésicale a beaucoup augmenté à la suite de la mise en eau du lac. Selon elle, en un an chez les garçons d'âge scolaire, il est passé par exemple de $9 \%$ à $99 \%$ à Amète et de $38 \%$ à $99 \%$ à Ampen (Ibidem). Toutes les informations épidémiologiques concernant les villages des rives du lac font ressortir une progression d'un ordre de grandeur comparable. La montée de la maladie suit fidèlement l'extension de la surface du lac.

De même à propos du barrage réservoir de Kainji au Nigeria, trois enquêtes réalisées en 1970, 1971, 1972 ont révélé une très forte augmentation de la prévalence de la bilharziose vésicale dans les villages environnants. Les résultats étaient particulièrement graves pour les groupes des jeunes de 15 à 21 ans, chez qui le taux était passé de $37 \%$ à 95\% (Ibidem 775).
Des exemples du même genre sont rapportés dans des zones où se pratique l'irrigation à grande échelle. Au Zimbabwe, on a observé l'impact de $l^{\prime}$ irrigation en comparant des taux $d$ 'incidences enregistrés dans deux zones, dont l'une se caractérisait par la culture en sec et l'autre par la culture en irriguée. Il ressort que dans les zones où se pratique la culture irriguée le taux de la bilharziose a été deux fois plus élevé que dans celles non irriguées (Ibidem).

Pour cette auteure, les individus atteints sont incapables d'effectuer des travaux pénibles même en l'absence des symptômes caractérisés. En cas de manifestations cliniques (Hématurie, diarrhée hémorragique) et complications graves, les malades peuvent être affectés d'une incapacité totale et la maladie peut même leur être fatale (Ibidem).

Quant au paludisme, on peut constater que les zones marécageuses situées aux alentours des lacs artificiels et des canaux d'irrigation constituaient un milieu favorable à la reproduction des anophèles et partant à la propagation du paludisme. Les manifestations cliniques du paludisme se caractérisent par l'excès des fièvres et la diminution du sang. Cette maladie fait de nombreuses victimes en Afrique.

On peut donc conclure que l'eau stoquée dans les réservoirs des barrages ne présente pas seulement des avantages. Tout en jouant un rôle capital dans la promotion du développement, elle peut aussi constituer un obstacle à celui-ci en créant des conditions défavorables à la santé des hommes qui sont censés travailler pour le même développement.

Les crises sanitaire et hygiénique occasionnent la désertion massive des régions dévastées par des catastrophes physiques ou d'autres facteurs qui entravent l'accès aux ressources économiques. Cette désertion est responsable des mouvements migratoires des régions pauvres vers les régions riches.

\section{La crise de l'environnement $\&$ du développement $\&$ ses implications sur le mouvement migratoire}

Selon Amadou Ba $(1994,6)$, il existe plusieurs façons par lesquelles la santé de la population influe sur la migration. En retour, la migration elle-même est un facteur dynamique dans le changement de l'état sanitaire. Pour cet auteur, on pourrait à la limite considérer que tout type de migration est de manière directe ou indirecte associée à la santé et réciproquement et affirme également que les conditions de santé des populations affectent directement ou indirectement le schéma de mobilité.

Depuis plus de trois décennies, les victimes de la crise de l'environnement et du développement se comptent par milliers. La dégradation de l'environnement écologique provoquée ou non est grandement responsable du déplacement massif de la population. "Pendant que le HCR estimait qu'ils y avaient plus de 15 millions des réfugiés au sens de la convention de Genève, ils $y$ avaient aussi plus de 10 millions des migrants qui sont considérés comme des réfugiés de l'environnement" (Jacobson 1988, 6).

En effet, quand le terroir familial est surexploité, la terre érodée, les occupants n'ont pas d'autres choix que $d$ aller chercher une terre fertile ailleurs. C'est par exemple le cas de 1 500 familles éthiopiennes qui ont été déplacées dans la région du Sud en 1984 lors de la grande famine qui a frappé dans ce pays. C'est aussi le cas de milliers de somaliens déplacés du nord du pays pendant la sécheresse de 1973-1974 pour être réinstallées dans les villages de Dujuuma, Sbalaale et Kunturwaarey près des rivières Shabaelle and Juba. Ces populations qui par la suite ont déserté les villages pour une destination incertaine font ainsi partie des victimes de la dégradation écologique.

Outreles problèmes environnementaux, les projets de développement forcent aussi la population à émigrer. C'est le cas des nomades Afar qui évincés de leurs traditionnelles terres par 
des irrigations pour la production de la culture de rente se sont vus obligés de se déplacer vers des terres moins fertiles.

Un autre exemple est celui du projet d'irrigation "Awash" qui consista en l'irrigation de terres pour la production du coton et du sucre dans la vallée de la rivière Awash. C'est ainsi que les terres fréquentées particulièrement par des nomades Afar furent transformées en terres pour la production de cultures de rente.

Dans le même ordre d'idée de dépossession des terres par les projets de développement, on peut citer le cas des sinistrés de grands barrages. En effet, les aménagements de grands barrages entraînent souvent le déplacement massif des populations. Ces déplacements s'expliquent notamment par l'implantation d'immenses lacs de retenue. C'est ainsi par exemple, que le barrage d'Assouan en Egypte provoqua le déplacement de $120000 \mathrm{Nu}$ biens, celui d'Akossombo au Ghana concerna 80000 personnes de différentes ethnies, tandis que le barrage de Kainji affecta 44000 personnes. Le barrage de Kossou en Côte d'Ivoire n'échappe pas à la règle. Sa construction a entraîné l'expulsion de 75.000 cultivateurs Baoulés (Lassailly 1980, 48).

Le déguerpissement de la population soudanaise en 1969, évincée par les inondations dues à la construction du grand barrage d'Assouan en Égypte, dont les eaux du lac artificiel baptisé "lac Nasser" s'étendent jusque sur le territoire soudanais est aussi un exemple de projet de développement, parmi tant d'autres, qui illustre bien le phénomène de dépossession et d'appauvrissement des paysans. "Cela commence par l'expropriation forcée des terres jugées nécessaires à l'implantation du projet et par l'expulsion parfois violente de ses occupants qui sont catapultés sur une trajectoire désastreuse" (Schwarz 1989). Plusieurs d'entre eux prennent le chemin des centres urbains et ainsi commence l'exode rural.

De nombreux travaux sur l'exode soulignent que le départmassif vers les villes doit être interprété en fonction dú fossé qui se creuse entre les ruraux et les citadins, ainsi que l'insuffisance des revenus paysans. En effet, la paupérisation du monde paysan due à l'affaiblissement des revenus agricoles et la destruction du cadre de vie à la campagne condamne les paysans à l'exode. Celui-ci associé au taux de natalité élevé explique l'explosion démographique et l'urbanisation anarchique que connaissent les villes africaines.

Sur ce dernier aspect, Vehasselt fait remarquer que l'explosion urbaine dans les pays tropicaux a crée des conditions écologiques favorables pour le développement des maladies endémiques, particulièrement celles engendrées par les moustiques. Selon le même auteur, cette situation est particulièrement frappante dans les quartiers périphérique ou s'établissent généralement les migrants d'origine rurale dont la résistance contre les parasites de type urbain est assez faible dans les premières années d'installation.

Enfin, les maladies provoquées par les perturbations écologiques dont les migrants sont en partie responsables ainsi que l'absence des services de santé adéquats constituent des facteurs déterminants dans la prise de décision de migrer.

\section{Conclusion}

Au terme de cet article, il nous est difficile d'appuyer sans réserve les études qui laissent croire que la dégradation de l'environnement en Afrique est due uniquement aux calamités naturelles et à l'explosion démographique. La cause principale de la crise de l'environnement est le mauvais développement qui crée un déséquilibre entre $l^{\prime}$ homme et son milieu naturel et accélère la prolétarisation de la population de la base. Ceci entraîne au fil des ans une pauvreté chronique qui se réflète aujourd'hui sur l'environnement écologique dont l'une des conséquences est la détérioration de la santé. Celle-ci associée à la crise alimentaire sont responsables des mouvements migratoires que connaissent les pays africains.

\section{Bibliographie}

Agence Canadienne de Développement International (ACDI). 1987. Environnement et développement, Politiques de l'ACDI, Hull.

Amadou, Ba. 1994. Revue de littérature sur le thème migration et santé. Montréal: Université de Montréal.

Catinot, René. 1984. "Afrique francophone, l'avenir forestier tropical se jouera dans le cadre du monde rural," dans Revue Bois et Forêts des tropiques, No. 203.

Commission Mondial pour l'Environnement et le Développement (CMED). 1989. Rapport Brundtland.

Centre de Recherche pour le Développement International (CRDI). 1992. Pour l'amour de la terre, Rapport de la Commission sur les pays en développement et les changements de l'environnement planétaire. Québec: Méridien.

Ela, Jean Marc. 1983. La ville en Afrique. Paris: Karthala.

Euzéby, Chantal. 1979. "La bilharziose et le développement", dans Revue du Tiers Monde, Vol. 20.

GERPE. 1990. "La coopération interafricaine en matière d'environnement", Québec, Cahiers du GERPE no. 2.

Galtung, Johan. 1977. Les stratégies des besoins fondamentaux. Paris: P.U.F.

Jacobson, J. 1988. "Environmental Refugees: A Yardstick of Hability." Washington DC: World Watch Institute.

Lassailly, Véronique. 1980. L'espace utile et transfert de la population en amont du barrage Kossou. Paris: Karthala.

Sachs, Ignacy. 1980. Stratégie de l'écodéveloppement. Paris: les éd. ouvrières.

Simard, P. and D. Diarra. 1993. “Discours sur l'environnement et stratégie de développement: points de vue du Nord et du Sud". Centre Sahel, Université Laval.

Soyed, A. M. 1975. "La faim dans le monde. Y-a-t-il une solution à la crise", le courrier, UNESCO, Mai.

Sukhatme, P. V. 1976. "Les ressources alimentaires mondiales", in Etudes de la FAO surl'alimentation et la population: Collection $F A O$, Développement économique et sociale, Rome.

UNICEF. 1992. Rapport annuel.

Thurnbull, Colin. 1962. The Forest People. New York: The American Museum of Natural History. $\square$

Refuge-Canada's Periodical on Refugees is now published six times a year.

(C) Shally B. Gachuruzi, 1996. Cette œuvre en libre accès fait l'objet d'une licence Creative Commons Attribution-NonCommercial 4.0 International License, laquelle autorise l'utilisation, la reproduction et la distribution de l'œuvre sur tout support à des fins non commerciales, pourvu que l'auteur ou les auteurs originaux soient mentionnés et que la publication originale dans Refuge : revue canadienne sur les réfugiés soit citée. 